\title{
Globular Clusters in the Galactic Bulge
}

\author{
E. Bica ${ }^{1}$, S. Ortolani ${ }^{2,3}$ and B. Barbuy ${ }^{4,5}$ \\ ${ }^{1}$ Departamento de Astronomia, Universidade Federal do Rio Grande do Sul, Av. Bento Gonçalves 9500, Porto Alegre 91501-970, Brazil \\ ${ }^{2}$ Dipartimento di Fisica e Astronomia, Università di Padova, I-35122 Padova, Italy \\ ${ }^{3}$ INAF-Osservatorio Astronomico di Padova, Vicolo dell'Osservatorio 5, I-35122 Padova, Italy \\ ${ }^{4}$ Universidade de São Paulo, IAG, Rua do Matão 1226, Cidade Universitária, São Paulo 05508-900, Brazil \\ ${ }^{5}$ Email: barbuy@astro.iag.usp.br
}

(Received September 4, 2015; ACCEPTED October 22, 2015)

\begin{abstract}
A view of the Galactic bulge by means of their globular clusters is fundamental for a deep understanding of its formation and evolution. Connections between the globular cluster and field star properties in terms of kinematics, orbits, chemical abundances, and ages should shed light on different stellar population components. Based on spatial distribution and metallicity, we define a probable best list of bulge clusters, containing 43 entries. Future work on newly discovered objects, mostly from the VVV survey, is suggested. These candidates might alleviate the issue of missing clusters on the far side of the bulge. We discuss the reddening law affecting the cluster distances towards the centre of the Galaxy, and conclude that the most suitable total-to-selective absorption value appears to be $R_{\mathrm{V}}=3.2$, in agreement with recent analyses. An update of elemental abundances for bulge clusters is provided.
\end{abstract}

Keywords: galaxy: bulge - galaxy: globular clusters

\section{INTRODUCTION}

The central parts of our Galaxy were prospected by Baade (1946), in order to detect its nucleus, and to have an indication of the morphological type of our Galaxy. The so-called Baade's Window was revealed, and from variable stars identified in the field, the bulge stellar population was identified to be similar to that defined in Baade (1944) as population II. In the early 60s, the notion of a Galactic bulge was already established (e.g. Courtes \& Cruvellier 1960). McClure (1969) found that the bulge stars were super metal-rich compared to the stars nearby the Sun. Whitford and Rich (1983) confirmed the high metal content of bulge stars from individual star spectroscopy.

Catalogues prepared along the decades show a steady growth of overall samples of globular clusters (hereafter referred to as GCs), together with photometric and spectroscopic information. Cannon (1929) used the Harvard plate spectra to give integrated spectral types of 45 GCs. Mayall (1946) measured radial velocities of $50 \mathrm{GCs}$, and the integrated spectral types of 40 of them. Kinman (1959) listed 32 GCs with indication of metallicity from their integrated spectra; Morgan (1959) directed efforts on a relatively bright sample of 13 bulge GCs, now known to be metal-rich.

Surveys with Schmidt plates since the 60s provided the Palomar and ESO star clusters, among others. Terzan (1968 and references therein) significantly increased the number of central GCs by reporting new faint ones in the bulge direction. These discoveries provided an important sample of GCs in the bulge.

Other studies of GC overall samples were presented by van den Bergh (1967), Zinn (1985 and references therein), Bica \& Pastoriza (1983). Webbink (1985) provided a catalogue of 154 GCs and candidates, with an important impact on subsequent observational efforts. More recently, Harris (1996, updated in 2010, hereafter Harris10 $)^{1}$ reports properties for 157 GCs.

It is interesting to see how the notion of GCs pertaining to the bulge and their spatial distribution evolved in the last decades. Frenk \& White (1982) found evidence that metalrich GCs formed a bar-like structure. Based on metallicity, scale height, and rotational velocities available at that time, Armandroff (1989 and references therein) interpreted a sample of low Galactic latitude metal-rich GCs as belonging to a disk system. Minniti (1995) instead, from metallicity and kinematics of GCs in the central $3 \mathrm{kpc}$ (about 20 $0^{\circ}$ ) from the Galactic centre, found evidence for these GCs to belong to the bulge. Côté (1999) derived metallicities and radial velocities

\footnotetext{
${ }^{1}$ www.physics.mcmaster.ca/ $\sim$ harris/mwgc.dat
} 
from high-resolution spectroscopy for a significant sample of GCs within $4 \mathrm{kpc}$ from the Galactic centre. He concluded that metal-rich GCs are associated with the bulge/bar rather than the thick disk.

An important step in the understanding of the nature of the bulge GCs was presented by Ortolani et al. (1995), where the metal-rich GCs NGC 6528 and NGC 6553 were found to have an old age, identical to the bulk of the bulge field stars and comparable to that of the halo clusters. Barbuy et al. (1998) derived new results and summarised the properties of $17 \mathrm{GCs}$ in the bulge projected within $5^{\circ}$ of the Galactic centre. They concluded that these clusters shared comparable properties with the bulge field stars, including not only metal-rich GCs but also intermediate metallicity ones. They also found that there are no clusters in a strip $2.8^{\circ}$ wide centred at about $0.5^{\circ}$ south of the Galactic plane.

Several comprehensive recent reviews have addressed the stellar populations, both field and GCs, in the Galactic bulge (e.g. Harris 2001; Rich 2013; Gonzalez \& Gadotti 2016). These reviews address also comparisons of the Galactic bulge with external galaxies.

In the following, we describe recent advances on detailed chemical abundances, distances, kinematical properties, and hints on possible association with subsystems in the central region of the Galaxy. We also prepare for the future by defining a bulge GC sample, which includes suggestions for future studies, in terms of unstudied objects. In particular, it is clear that proper motion derivation is still needed for most GCs.

\section{THE SAMPLE OF BULGE GLOBULAR CLUSTERS}

In the past, angular distances from the Galactic centre were the basis for selecting bulge GCs in the central parts of the Galaxy (e.g. Zinn 1985; Barbuy et al. 1998).

As a first approach, we selected clusters with angular distances below $20^{\circ}$. Our next step was to use the Galactocentric distances of the clusters, given that now they are more reliable - see Harris 10. We adopted a distance to the Galactic centre of $8 \mathrm{kpc}$ (Bobylev et al. 2014; Reid et al. 2014). For selecting the clusters, we have experimented different distances from the Galactic centre of 6, 5, 4, and $3 \mathrm{kpc}$. In each case, we checked for bulge clusters and halo intruders. We concluded that a cut-off of $3 \mathrm{kpc}$ is best in terms of isolating a bona-fide bulge cluster sample, with little contamination. We finally applied a metallicity filter: Zoccali et al. (2008), Hill et al. (2011), Ness et al. (2013a), and Rojas-Arriagada et al. (2014) have shown that the lower metallicity end of the bulge is around $[\mathrm{Fe} / \mathrm{H}]=-1.3$. This is confirmed with the findings by Walker and Terndrup (1991), Lee (1992), Dékány et al. (2013), Pietrukowicz et al. (2012, 2015), and Gran et al. (2016), Dékány et al. (2013) and Lee (1992), all of them having demonstrated that there is a peak of RR Lyrae with $[\mathrm{Fe} / \mathrm{H}] \approx-1.0$, centrally concentrated and spheroidal (except for Pietrukowicz et al. 2015 that found it to be elongated), corresponding to an old bulge. We verified that GCs with $[\mathrm{Fe} / \mathrm{H}]<-1.5$ corresponded to well-known halo clusters in most cases, and we excluded GCs with $[\mathrm{Fe} / \mathrm{H}]<-1.5$.

Criteria using the space velocity $V$, as proposed by Dinescu, Girard, \& van Altena (1997), Dinescu, van Altena, \& Girard (1999a), Dinescu, Girard, \& van Altena (1999b), Dinescu et al. (2003), and Casetti-Dinescu et al. (2007, 2010, 2013), are only feasible if proper motions are available, besides radial velocities. In particular, Dinescu et al. (2003) verified the classification of clusters as members of different galaxy components in terms of kinematics. The use of this criterion is possible for about a third of the GCs, at the present stage. Gaia results in a few years will bring higher precision, and new data for a number of GCs . A few groups are applying proper motion cleaning to bulge clusters (e.g. Dinescu et al. 2003; Rossi et al. 2015). Therefore, Carretta et al. (2010) criterion of space velocity $\mathrm{V}$ values to select stellar populations, is not possible presently for having only the radial velocity in many cases, but may be applicable in a few years.

In Table 1, we present the selected bulge clusters, following the criteria explained above. Djorgovski 1 is added to the list, because it was in the Barbuy et al. (1998)'s list, and the distance available in Harris10, based on infrared data, is probably overestimated. The clusters are ordered by right ascension, to be compatible with Harris 10.

The distances from the Sun are from Harris10 mainly based on the visual magnitude of the HB, while Valenti, Ferraro, \& Origlia (2007) give distances based on isochrone fitting in $J H K$. We also report distances derived from the Galactic centre, as given by Harris10. For Kronberger 49, not listed in Harris 10, data are from Ortolani et al. (2012). The distance derivation is crucial in order to get the position of the clusters relative to the Galactic centre and the bar. In most cases, for a cluster located inside the bar, its orbit is trapped, given the high mass of the bar. In fact, more than half the bulge mass is in the bar, and for this reason, the bulge clusters that show low kinematics are probably trapped (Rossi et al. 2015). Given the old age of the clusters, it is likely that they were formed before the bar, and later trapped. The dynamical behaviour of such clusters was illustrated for HP 1 (Ortolani et al. 2011), and for nine GCs by Rossi, Ortolani, Casotto, Barbuy, and Bica (in preparation), all of them showing a low maximum height.

\subsection{Intruders and missed bulge clusters?}

In Table 2, we list possible intruders to our main selection, as well as limiting cases, and we collected a few more GCs with other distance and metallicity criteria. Subsamples are classified as follows:

(a) Probable halo intruders with $[\mathrm{Fe} / \mathrm{H}]<-1.5$ : we found $6 \mathrm{GCs}$ with distances to the Galactic centre smaller than $3 \mathrm{kpc}$ (our selected bulge volume), but with metallicity lower than $[\mathrm{Fe} / \mathrm{H}]<-1.5$, and besides with very high 
Table 1. Bulge globular clusters. Reddening values in columns 4, 5, and 6 correspond to Harris10, Valenti et al. (2007), and our studies along the years. Galactocentric distances and metallicities are from Harris10, except for UKS 1 (see Section 3). References: 1: CasettiDinescu et al. 2010; 2: Ortolani et al. 2011; 3: Rossi et al. 2015; 4: Casetti-Dinescu et al. 2013; 5: Dinescu et al. 2003; 6: Zoccali et al. 2001; 7: Cudworth \& Hanson 1993. Notes: *sample from Barbuy et al. 1998.

\begin{tabular}{|c|c|c|c|c|c|c|c|c|c|c|c|}
\hline Name & $1\left(^{\circ}\right)$ & $\mathrm{b}\left({ }^{\circ}\right)$ & $\begin{array}{c}E(B-V) \\
\text { Harris }\end{array}$ & $\begin{array}{c}E(B-V) \\
\quad \text { Valenti }\end{array}$ & $\begin{array}{c}E(B-V) \\
\text { Ortolani }\end{array}$ & $\begin{array}{c}\mathrm{d}_{\odot}^{\text {Harris }} \\
(\mathrm{kpc})\end{array}$ & $\begin{array}{l}\mathrm{d}_{\odot}^{\text {Valenti }} \\
(\mathrm{kpc})\end{array}$ & $\begin{array}{c}\mathrm{d}_{\mathrm{GC}} \\
(\mathrm{kpc})\end{array}$ & {$[\mathrm{Fe} / \mathrm{H}]$} & $\mathrm{km} \mathrm{s}^{v_{\mathrm{r}}}$ & $\mathrm{km} \mathrm{s}^{v_{\mathrm{s}}}$ \\
\hline Terzan 3 & 345.08 & 9.19 & 0.73 & 0.73 & 0.72 & 8.20 & 8.10 & 2.5 & -0.72 & -136.3 & \\
\hline ESO452-SC11 & 351.91 & 12.10 & 0.46 & - & 0.58 & 8.30 & - & 2.1 & -1.50 & - & \\
\hline NGC 6256 & 347.79 & 3.31 & 1.09 & 1.20 & 1.10 & 10.30 & 9.10 & 3.0 & -1.02 & -101.4 & \\
\hline NGC 6266 (M 62) & 353.58 & 7.32 & 0.47 & 0.47 & - & 6.80 & 6.60 & 1.7 & -1.18 & -70.1 & \\
\hline NGC 6304 & 355.83 & 5.38 & 0.54 & 0.58 & 0.50 & 5.90 & 6.00 & 2.3 & -0.45 & -107.3 & $195.69^{5}$ \\
\hline NGC 6316 & 357.18 & 5.76 & 0.54 & 0.56 & - & 10.40 & 11.60 & 2.6 & -0.45 & 71.4 & $128.38^{5}$ \\
\hline NGC 6325 & 0.97 & 8.00 & 0.91 & - & 0.95 & 7.80 & - & 1.1 & -1.25 & 29.8 & \\
\hline NGC 6342 & 4.90 & 9.73 & 0.46 & 0.57 & - & 8.50 & 8.40 & 1.7 & -0.55 & 115.7 & $155.26^{1}$ \\
\hline NGC 6355 & 359.58 & 5.43 & 0.77 & 0.81 & 0.78 & 9.20 & 9.00 & 1.4 & -1.37 & -176.9 & \\
\hline Terzan 2* (HP 3) & 356.32 & 2.30 & 1.87 & 1.87 & 1.54 & 7.50 & 7.40 & 0.8 & -0.69 & 109.0 & $141.87^{3}$ \\
\hline Terzan 4* (HP 4) & 356.02 & 1.31 & 2.00 & 2.05 & 2.35 & 7.20 & 6.70 & 1.0 & -1.41 & -50.0 & $172.17^{3}$ \\
\hline HP $1^{*}$ (BH 229) & 357.42 & 2.12 & 1.12 & 1.18 & 1.19 & 8.20 & 6.80 & 0.5 & -1.00 & 45.8 & $241.45^{2}$ \\
\hline Liller $1^{*}$ & 354.84 & -0.16 & 3.07 & 3.09 & 3.05 & 8.20 & 7.90 & 0.8 & -0.33 & 52.0 & \\
\hline Terzan $1^{*}$ (HP 2) & 357.57 & 1.00 & 1.99 & 1.99 & 1.67 & 6.70 & 6.60 & 1.3 & -1.03 & 114.0 & $125.36^{3}$ \\
\hline Ton 2 (Pis 26) & 350.80 & -3.42 & 1.24 & - & 1.26 & 8.20 & - & 1.4 & -0.70 & -184.4 & \\
\hline NGC 6401 & 3.45 & 3.98 & 0.72 & 1.10 & 0.53 & 10.60 & 7.70 & 2.7 & -1.02 & -65.0 & \\
\hline VVV-CLO02 & 359.56 & 0.89 & 2.88 & - & - & 7.3 & - & 0.7 & -0.40 & - & \\
\hline Pal 6* & 2.09 & 1.78 & 1.46 & - & 1.33 & 5.80 & - & 2.2 & -0.91 & 181.0 & $219.67^{3}$ \\
\hline Djorg $1^{*}$ & 356.67 & -2.48 & - & - & - & - & - & & - & - & \\
\hline Terzan $5^{*}$ & 3.81 & 1.67 & 2.28 & 2.38 & 2.49 & 6.90 & 5.90 & 1.2 & -0.23 & -93.0 & \\
\hline NGC 6440 & 7.73 & 3.80 & 1.07 & 1.15 & 1.00 & 8.50 & 8.20 & 1.3 & -0.36 & -76.6 & \\
\hline Terzan 6* (HP 5) & 358.57 & -2.16 & 2.35 & 2.35 & 2.24 & 6.80 & 6.70 & 1.3 & -0.56 & 126.0 & \\
\hline Terzan $9 *$ & 3.60 & -1.99 & 1.76 & 1.79 & 1.95 & 7.10 & 5.60 & 1.1 & -1.05 & 59.0 & $126.35^{3}$ \\
\hline ESO456-SC38* (Djorg 2) & 2.76 & -2.50 & 0.94 & 0.94 & 0.89 & 6.30 & 7.00 & 1.8 & -0.65 & - & \\
\hline Terzan $10^{*}$ & 4.42 & -1.86 & 2.40 & - & 2.48 & 5.80 & - & 2.3 & -1.00 & - & \\
\hline NGC $6522^{*}$ & 1.02 & -3.93 & 0.48 & 0.66 & 0.55 & 7.70 & 7.40 & 0.6 & -1.34 & -21.1 & $140.61^{3}, 235.60^{5}$ \\
\hline NGC 6528* & 1.14 & -4.17 & 0.54 & 0.62 & 0.55 & 7.90 & 7.50 & 3.0 & -0.11 & 206.6 & $198.75^{5}$ \\
\hline NGC 6539 & 20.80 & 6.78 & 1.02 & 1.08 & - & 7.80 & 8.40 & 3.0 & -0.63 & 31.0 & \\
\hline NGC 6540* (Djorg 3) & 3.29 & -3.31 & 0.66 & 0.66 & 0.60 & 5.30 & 5.20 & 2.8 & -1.35 & -17.7 & $157.83^{3}$ \\
\hline NGC 6553* & 5.25 & -3.02 & 0.63 & 0.84 & 0.63 & 6.00 & 4.90 & 2.2 & -0.18 & -3.2 & $225.61^{5}, 230^{6}$ \\
\hline NGC 6558 & 0.20 & -6.03 & 0.44 & - & 0.38 & 7.40 & - & 1.0 & -1.32 & -197.2 & $188.66^{3}$ \\
\hline Kronberger 49 & 7.63 & -2.01 & - & - & 1.35 & - & - & 1.19 & -0.10 & - & - \\
\hline AL 3* (BH 261) & 3.35 & -5.26 & 0.36 & - & 0.36 & 6.50 & - & 1.7 & - & - & \\
\hline GLIMPSE02 & 14.13 & -0.64 & 7.85 & - & - & 5.50 & - & 3.0 & -0.33 & - & \\
\hline Mercer 5 & 17.59 & -0.86 & - & - & - & - & - & 2.45 & -0.86 & - & - \\
\hline NGC 6624 & 2.79 & -7.91 & 0.28 & 0.28 & - & 7.90 & 8.40 & 1.2 & -0.44 & 53.9 & \\
\hline NGC 6626 (M 28) & 7.80 & -5.58 & 0.40 & - & - & 5.50 & - & 2.7 & -1.32 & 17.0 & $131.26^{4}, 75.07^{7}$ \\
\hline NGC 6638 & 7.90 & -7.15 & 0.41 & 0.43 & - & 9.40 & 10.30 & 2.2 & -0.95 & 18.1 & \\
\hline NGC 6637 (M 69) & 1.72 & -10.27 & 0.18 & 0.14 & - & 8.80 & 9.40 & 1.7 & -0.64 & 39.9 & \\
\hline NGC 6642 & 9.81 & -6.44 & 0.40 & 0.60 & 0.42 & 8.10 & 8.60 & 1.7 & -1.26 & -57.2 & \\
\hline NGC 6652 & 1.53 & -11.38 & 0.09 & - & 0.10 & 10.00 & - & 2.7 & -0.81 & -111.7 & \\
\hline NGC 6717 (Pal 9) & 12.88 & -10.90 & 0.22 & - & 0.23 & 7.10 & - & 2.4 & -1.26 & 22.8 & \\
\hline NGC 6723 & 0.07 & -17.30 & 0.05 & - & - & 0.87 & - & 2.6 & -1.10 & -94.5 & $171.11^{5}$ \\
\hline
\end{tabular}

spatial velocities (Dinescu et al. 1999a; Casetti-Dinescu et al. 2010), all pointing to perigalactic locations of halo GCs.

(b) Outer bulge shell with distances $3<\mathrm{R}<4.5 \mathrm{kpc}$ : this surrounding bulge shell does probably contain true bulge GCs, that should be near apogalacticon. Interestingly, the few space velocities available (Dinescu et al. 1999a, Cudworth \& Hanson 1993) for this sample are rather low. (c) Outer bulge shell intruders: five GCs are located in this shell (b), but showing low metallicities, as for example M22, having also a high velocity typical of halo, like in group (a).

(d) Metal-rich GCs $([\mathrm{Fe} / \mathrm{H}]>-1.0)$ beyond $\mathrm{R}>4.5 \mathrm{kpc}$ : this sample corresponds to the ones that conveyed the idea of 'disk GCs' (e.g. Armandroff 1989). Many of these key and well-known GCs do not have space velocities derived so far. This would be of great interest to 
Table 2. Intruders and missed bulge clusters? (a) Possible halo intruders with $[\mathrm{Fe} / \mathrm{H}]<-1.5$; (b) Shell: Distances $3<\mathrm{R}<4.5 \mathrm{kpc}$; (c) Intruders to shell; (d) $[\mathrm{Fe} / \mathrm{H}]>-1.0$ and $\mathrm{R}>4.5 \mathrm{kpc}$; (e) Intruders to disc; (f) no parameters enough; *looks halo intruder in the shell, despite distance; ${ }^{* *}$ see Section 3. References: 1: Casetti-Dinescu et al. 2010; 4: Casetti-Dinescu et al. 2013; 7: Cudworth and Hanson 1993; 8: Dinescu et al. 1997; 9: Casetti-Dinescu et al. 2007; 10: Casetti-Dinescu et al. 2013.

\begin{tabular}{|c|c|c|c|c|c|c|c|}
\hline Name & Name & $1\left(^{\circ}\right)$ & $\mathrm{b}\left({ }^{\circ}\right)$ & $\mathrm{d}_{\mathrm{GC}}(\mathrm{kpc})$ & {$[\mathrm{Fe} / \mathrm{H}]$} & $v_{\mathrm{r}} \mathrm{km} \mathrm{s}^{-1}$ & $v_{\mathrm{s}} \mathrm{km} \mathrm{s}^{-1}$ \\
\hline \multicolumn{8}{|c|}{ (a) Probable halo intruders with $[\mathrm{Fe} / \mathrm{H}]<-1.5$} \\
\hline NGC 6144 & & 351.93 & 15.70 & 2.7 & -1.76 & 201.9 & $366.70^{10}$ \\
\hline NGC 6273 & M 19 & 356.87 & 9.38 & 1.7 & -1.73 & 143.8 & $213.73^{1}$ \\
\hline NGC 6287 & & 0.13 & 11.02 & 2.1 & -2.10 & -279.1 & $280.19^{1}$ \\
\hline NGC 6293 & & 357.62 & 7.83 & 2.3 & -1.99 & -137.4 & $201.35^{1}$ \\
\hline NGC 6333 & M9 & 5.54 & 10.70 & 1.7 & -1.77 & 239.8 & $284.63^{1}$ \\
\hline NGC 6541 & & 349.29 & -11.18 & 2.1 & -1.81 & -154.2 & \\
\hline \multicolumn{8}{|c|}{ (b) Outer Shell of distances $3<\mathrm{R}<4.5 \mathrm{kpc}$} \\
\hline Lynga 7 & & 328.77 & -2.79 & 4.3 & -1.01 & 8.0 & \\
\hline NGC 6171 & M107 & 3.37 & 23.01 & 3.3 & -1.02 & -23.0 & $87.01^{7}$ \\
\hline NGC 6235 & & 358.92 & 13.52 & 4.2 & -1.28 & 96.8 & \\
\hline NGC 6402 & M14 & 21.32 & 14.81 & 4.0 & -1.28 & -42.1 & \\
\hline NGC 6388 & & 345.56 & -6.74 & 3.1 & -0.55 & 84.2 & $43.30^{1}$ \\
\hline NGC 6352 & & 341.42 & -7.17 & 3.3 & -0.64 & -134.1 & \\
\hline NGC 6380 & Ton 1 & 350.18 & -3.42 & 3.3 & -0.75 & 2.1 & \\
\hline NGC 6441 & & 353.53 & -5.01 & 3.9 & -0.46 & 22.9 & $48.80^{1}$ \\
\hline NGC 6496 & & 348.02 & -10.01 & 4.2 & -0.46 & -108.4 & \\
\hline NGC 6517 & & 19.23 & 6.76 & 4.2 & -1.23 & -26.5 & \\
\hline NGC 6544* & & 5.84 & -2.20 & 5.1 & -1.4 & -17.7 & \\
\hline 2MASS-GC02 & & 9.78 & -1.08 & $3.9^{8}$ & -1.08 & -227.4 & \\
\hline IC 1276 & Pal 7 & 21.83 & 5.67 & 3.7 & -0.75 & 169.2 & \\
\hline Terzan 12 & & 8.36 & -2.10 & 3.4 & -0.50 & 104.2 & \\
\hline NGC 6569 & & 0.48 & -6.68 & 3.1 & -0.76 & -20.3 & \\
\hline NGC 6712 & & 25.35 & -4.32 & 3.5 & -1.02 & -94.7 & $166.55^{7}$ \\
\hline \multicolumn{8}{|c|}{ (c) Outer shell intruders } \\
\hline NGC 6139 & & 342.37 & 6.94 & 3.6 & -1.65 & 11.5 & \\
\hline NGC 6453 & & 355.72 & -3.87 & 3.7 & -1.50 & -76.7 & \\
\hline NGC 6535 & & 27.18 & 10.44 & 3.9 & -1.79 & -200.4 & \\
\hline NGC 6656 & M22 & 9.89 & -7.55 & 4.9 & -1.70 & -136.6 & $277.62^{4}, 187.27^{7}$ \\
\hline NGC 6809 & M55 & 8.80 & -23.27 & 3.9 & -1.94 & 181.8 & \\
\hline \multicolumn{8}{|c|}{ (d) Metal-rich clusters $([\mathrm{Fe} / \mathrm{H}]>-1.0)$ beyond $\mathrm{R}>4.5 \mathrm{kpc}$} \\
\hline NGC 104 & 47 Tuc & 305.90 & -44.89 & 7.4 & -0.72 & -26.7 & $77.76^{7}$ \\
\hline NGC 5927 & & 326.60 & 4.86 & 4.6 & -0.49 & -107.2 & $230.2^{9}$ \\
\hline BH 176 & & 328.41 & 4.34 & 12.9 & 0.00 & - & \\
\hline NGC 6356 & & 6.72 & 10.22 & 7.5 & -0.40 & 38.0 & $144.54^{1}$ \\
\hline NGC 6362 & & 325.55 & -17.57 & 5.1 & -0.99 & -15.6 & $144.55^{8}$ \\
\hline NGC 6366 & & 18.41 & 16.04 & 5.0 & -0.59 & -108.6 & \\
\hline UKS 1 & & 5.12 & 0.76 & $14.6^{* *}$ & -0.64 & 57.0 & \\
\hline Pfleiderer 2 & & 22.28 & 9.32 & 9.7 & 0.0 & - & \\
\hline Pal 8 & & 14.10 & -6.80 & 5.5 & -0.37 & -32.3 & \\
\hline NGC 6760 & & 36.11 & -3.92 & 4.8 & -0.40 & -13.2 & \\
\hline Pal 11 & & 31.81 & -15.58 & 6.7 & -0.40 & -56.0 & \\
\hline NGC 6838 & M71 & 56.74 & -4.56 & 6.7 & -0.78 & -7.5 & $96.88^{7}$ \\
\hline \multicolumn{8}{|c|}{ (e) intruders to (d) } \\
\hline NGC 6284 & & 358.35 & 9.94 & 7.5 & -1.26 & 36.7 & $249.56^{1}$ \\
\hline FSR 1767 & & 352.60 & -2.17 & 6.53 & -1.20 & - & - \\
\hline \multicolumn{8}{|c|}{ (f) Little studied clusters without enough parameters } \\
\hline $\begin{array}{l}\text { FSR } 1735 \\
\end{array}$ & & 339.20 & -1.85 & 3.7 & - & $\ldots$ & \\
\hline VVV-CL003 & & 358.40 & 0.73 & 13.0 & -0.10 & $\ldots$ & \\
\hline VVV-CL001 & & 5.27 & 0.78 & - & - & $\ldots$ & \\
\hline VVV-CL004 & & 6.79 & 1.72 & - & - & $\ldots$ & \\
\hline 2MASS-GC01 & & 10.47 & 0.10 & 4.5 & - & $\ldots$ & \\
\hline GLIMPSE-C01 & & 31.30 & -0.10 & 4.9 & - & $\ldots$ & \\
\hline
\end{tabular}



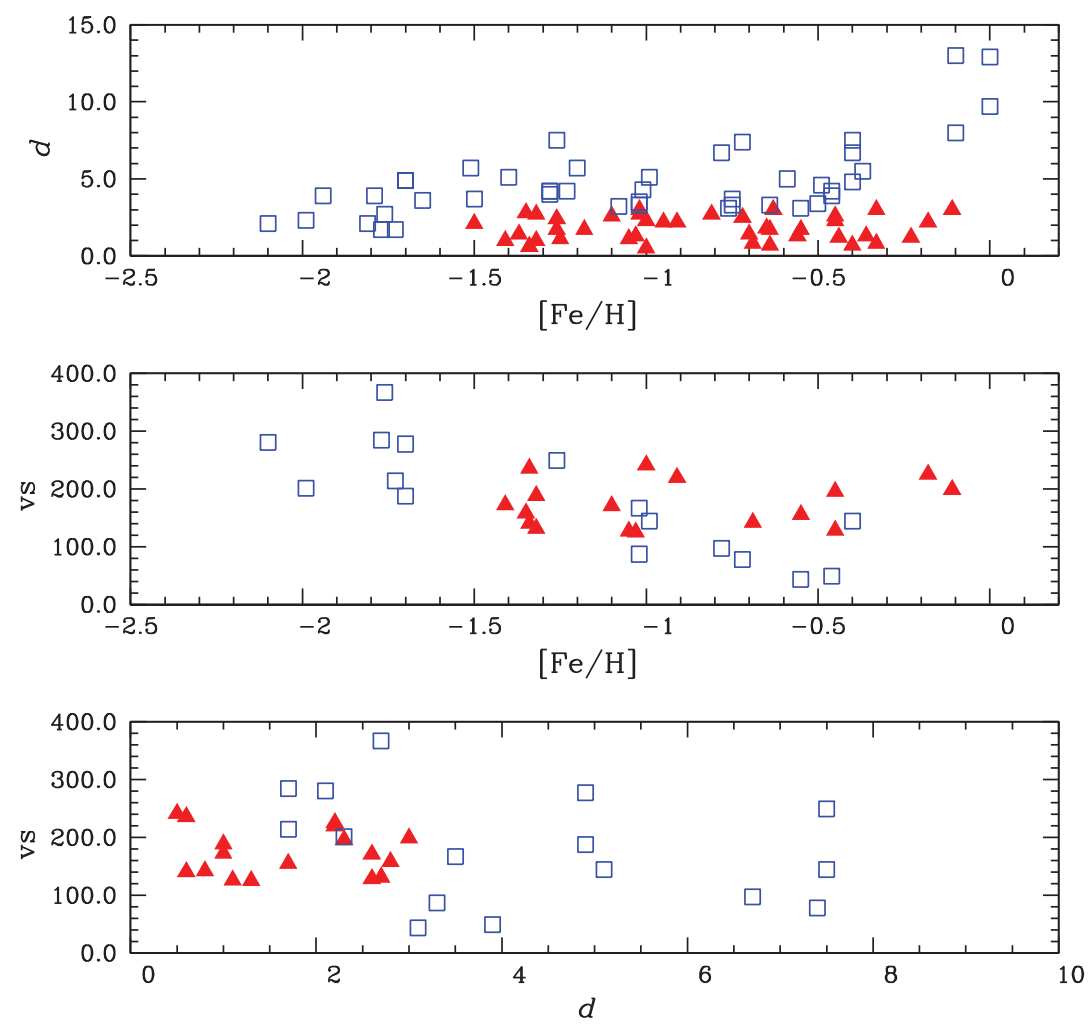

Figure 1. Comparisons between Galactocentric distances $\mathrm{d}(\mathrm{kpc})$, space velocities $\mathrm{V}_{\mathrm{s}}\left(\mathrm{km} \mathrm{s}^{-1}\right)$ and metallicity $[\mathrm{Fe} / \mathrm{H}]$. Symbols: red triangles: bulge clusters (Table 1), open squares: intruders or candidates (Table 2).

constrain the possibility to link them to the thick disk.

(e) Intruders to (d). Data for FSR 1767 are from Bonatto et al. (2007, 2009).

(f) Little studied GCs without enough parameters: probably key objects for future analysis (Section 6). In Figure 1 are plotted distances (kpc) vs. metallicity $[\mathrm{Fe} / \mathrm{H}]$, space velocity $\mathrm{V}_{\mathrm{S}}\left(\mathrm{km} \mathrm{s}^{-1}\right)$ vs. $[\mathrm{Fe} / \mathrm{H}]$ and vs. distance $(\mathrm{kpc})$, for the bulge sample (Table 1) and intruders (Table 2), for which such data are available.

\subsection{Multiple population clusters in the bulge}

Terzan 5 was identified to have at least two stellar populations and given its high mass, it was proposed to be a stripped dwarf galaxy (Ferraro et al. 2009; Origlia et al. 2013; Massari et al. 2014). Saracino et al. (2015) concluded that Liller 1 is as massive as Terzan 5 or $\omega$ Centauri. These massive clusters have absolute magnitudes around $M_{\mathrm{V}} \approx-10$.

On the other hand, the faintest bulge GCs are as faint as $M_{\mathrm{V}} \approx-4$, such as Terzan 9 and AL 3. These magnitudes are comparable to those of the ultra-faint galaxies (e.g. McConnachie 2012; Bechtol et al. 2015).

By multiple stellar populations, we refer to different metallicities $[\mathrm{Fe} / \mathrm{H}]$ and/or age in a same cluster. For most clusters, there are hints of two populations, but no difference in metallicity. A comprehensive discussion on $\mathrm{Na}-\mathrm{O}$ anticorrelation was given by Gratton et al. (2015), where differences be- tween red and blue horizontal branch (HB) stars allow to derive some important conclusions. See further discussions on multiple populations revealed by $\mathrm{Na}-\mathrm{O}$ anticorrelations in Section 4. Since this effect appears to be present for most clusters, this is incorporated in the definition of GCs, and it is not a concern in the present work.

\section{DISTANCES}

Distances of bulge GCs are mainly based on the HB luminosity level. The calculation of distances depends on three basic inputs: (1) the HB absolute magnitude, (2) the reddening, and (3) the reddening law. These three datasets slightly depend on the metallicity. The HB absolute magnitude may also depend on the He abundance. The current statistics is based mostly on the distances reported in Harris10, where the $\mathrm{HB}$ level is given by the relation: $\mathrm{M}(\mathrm{HB})=0.16[\mathrm{Fe} / \mathrm{H}]$ +0.84 . In Harris 10, if the HB level is not available, specific references are used. This relation is somewhat different from that used by Barbuy et al. (1998) in their catalogue of 17 inner bulge clusters, adopted from Jones et al. (1992): M(HB) = $0.16[\mathrm{Fe} / \mathrm{H}]+0.98$. This latter relation gives an $\mathrm{HB}$ level of about 0.14 mag fainter, producing as well smaller distance moduli, but this difference is negligible when compared to other uncertainties. It is also important to point out that the main difference between Harris10 and Barbuy et al. (1998) values, is that the latter was based on optical colour- 
magnitude diagrams (CMDs) only. The list of distances also makes use of the JHK CMDs when it is considered more reliable. Finally, the catalogue by Bica et al. (2006) uses basically the same assumptions as Harris 10 .

Reddening and reddening law: The reddening is a key parameter in the derivation of the distances based on the photometric technique. For most of the low latitude bulge GCs, an error of $5 \%$ in reddening produces a typical error of $\sim 15 \%$ in the absorption, which propagates with the same fraction to the distance modulus. In Harris 10, the reddening has been derived from Webbink (1985), Zinn (1985), and Reid et al. (1988). The standard reddening law $\left(R_{\mathrm{V}}=3.1\right)$ has been used to derive the visual absorption. Bica et al. (2006) used basically the same input values, but they converted $E(B-V)$ into $A_{\mathrm{V}}$ using $R_{\mathrm{V}}=3.1$ for clusters with $[\mathrm{Fe} / \mathrm{H}]<-1.0$ and $R_{\mathrm{V}}=3.6$ for $[\mathrm{Fe} / \mathrm{H}]>-1.0$ following Grebel and Roberts (1995), and $R_{\mathrm{V}}$ values have been interpolated in the intermediate metallicity interval. In principle, this choice should produce shorter distances than Harris10 for high metallicity clusters. A different computation was performed for most of the inner bulge clusters presented in Barbuy et al. (1998). Equation (A1) of Dean, Warpen, \& Cousins (1978) was used to convert $E(V-I)$ to $E(B-V)$ and then the reddening dependence of $R_{\mathrm{V}}$ on $E(B-V)$ as given in Olson (1975) has been used to convert $E(B-V)$ into $A_{\mathrm{V}}: R_{\mathrm{V}}=3.1+0.05([\mathrm{Fe} / \mathrm{H}])$.

Independent distances and reddening values have been obtained by Valenti et al. (2007) for 37 bulge clusters, using infrared $J H K$ photometry. The main advantage of the $J H K$ derived distances is that the reddening versus absorption ratio is almost constant even if $R_{\mathrm{V}}$ varies (Fitzpatrick 1999; Cardelli, Clayton, \& Mathis 1989). The comparison of the optical versus IR distances give a chance to probe the reddening law, in the optical regime, in the direction of the Galactic bulge.

Figure 2 shows the distance differences between different $R_{\mathrm{V}}$ values versus reddening. The absolute magnitude of the horizontal branch $\mathrm{M}(\mathrm{HB})$ is assumed to be of $\mathrm{M}(\mathrm{HB})$ $=0.68$ for $[\mathrm{Fe} / \mathrm{H}]=-1.0$, at $E(B-V)=0$. Therefore, at the distance of the Galactic centre, assumed here to be of $8 \mathrm{kpc}$, the distance modulus is $\mathrm{m}-\mathrm{M}=14.5$, and $\mathrm{m}(\mathrm{HB})=14.5$ $+0.68=15.18$. So for the zero point, we assume: $E(B-V)$ $=0, \mathrm{~m}(\mathrm{HB})=15.18$. We use 12 clusters in common with Valenti et al. (2007), and adding Terzan 9 (Ortolani, Bica, $\&$ Barbuy 1999). From this sample, an average distance difference of $\mathrm{d}(\mathrm{IR})-\mathrm{d}($ optical $)=0.65 \mathrm{kpc}$ has been derived. In the infrared sample, we have also three clusters (Terzan 4, Terzan 5, and NGC 6528) with data from Hubble Space Telescope (HST)/NICMOS (Ortolani et al. 2007) . In these cases, we adopted an average value between NICMOS and Valenti et al. (2007). At an average distance of $8 \mathrm{kpc}$, the $0.65 \mathrm{kpc}$ difference is equivalent to about $0.3 \mathrm{mag}$ in distance modulus. These differences between IR and optical distances as a function of reddening $E(B-V)$ are plotted in Figure 3. Conclusions from Figures 2 and 3 are: (a) it is clear that the optical data produce shorter distances, in particular at high reddening; (b) in order to get comparable infrared and opti-

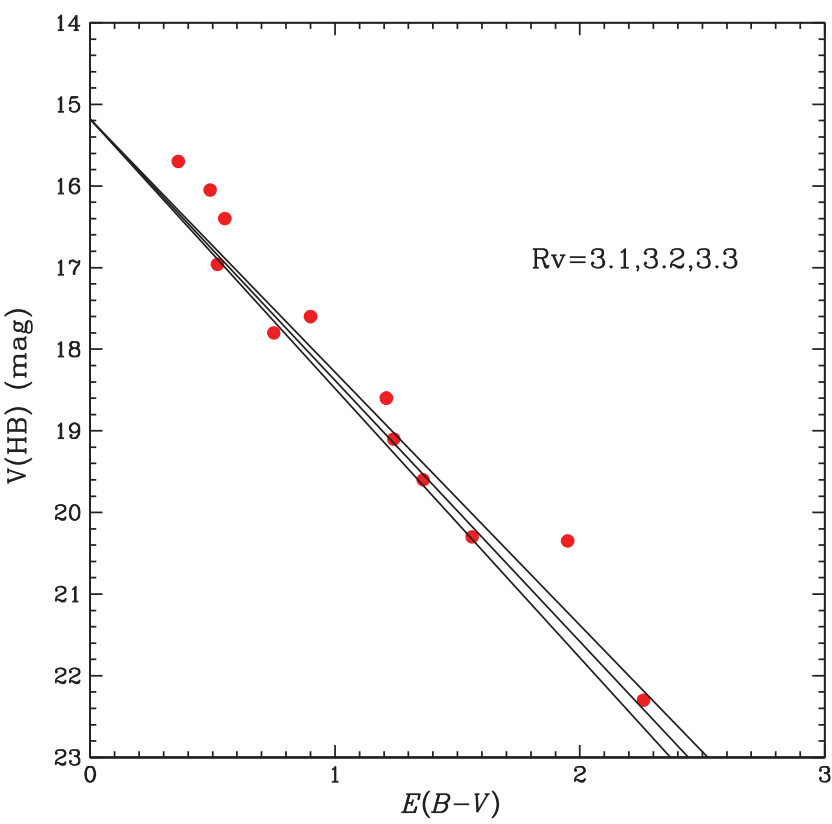

Figure 2. Horizontal branch magnitude $\mathrm{V}(\mathrm{HB})$ versus reddening $E(B-V)$ for the bulge clusters. Total-to-selective absorption $R_{\mathrm{V}}$ values are indicated. A distance to the Galactic centre of $8 \mathrm{kpc}$ is assumed.

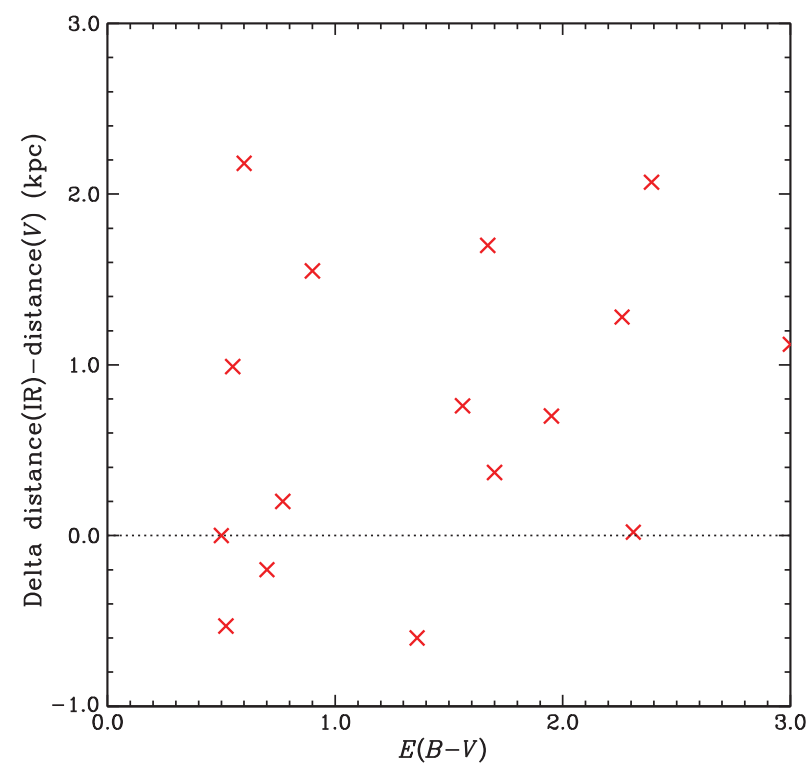

Figure 3. Difference of IR versus optical distances as a function of reddening for a sample of bulge clusters in common between Barbuy et al. (1998) and Valenti et al. (2007).

cal distances, we have to adopt an average total-to-selective absorption $R_{\mathrm{V}}=3.2$. This average value is slightly lower than that adopted in Barbuy et al. (1998) of $R_{\mathrm{V}}=3.39$ (see their Table 4), but it is still higher than the standard $R_{\mathrm{V}}=3.1$ value, and it is in agreement with recent studies of the reddening law in different conditions of reddening, metallicity, and intrinsic colours of the stars (Hendricks et al. 2012; McCall 2004). A further test can be performed following Racine 


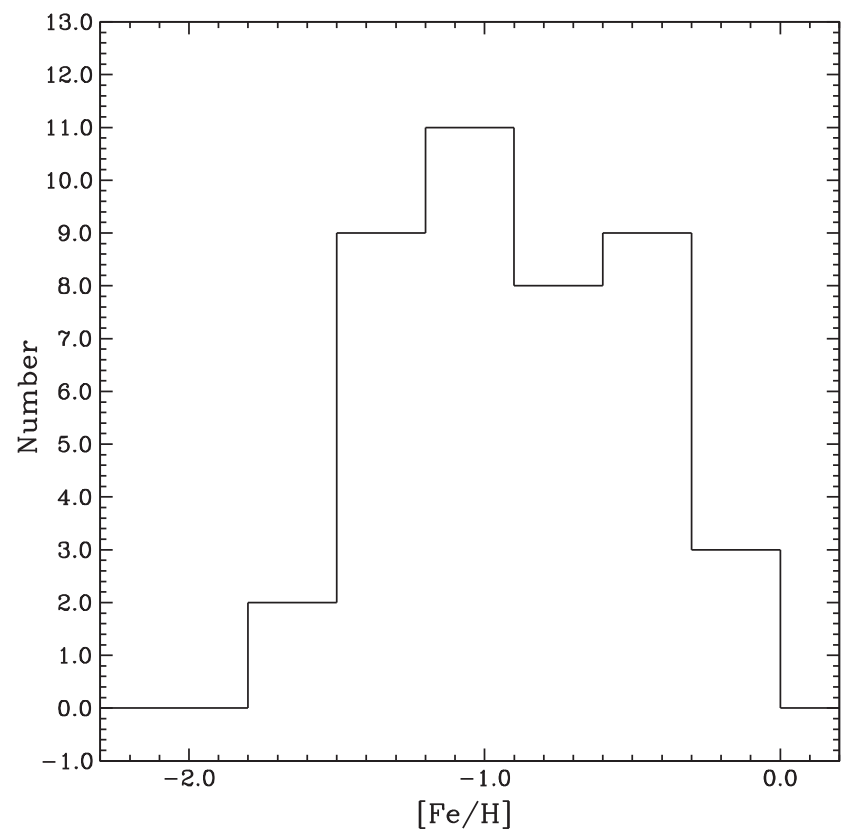

Figure 4. Metallicity histogram of sample bulge clusters (Table 1).

\& Harris (1989) and Barbuy et al. (1998), plotting $V(\mathrm{HB})$ as a function of reddening. Assuming that, on the average, the inner bulge clusters (within $3 \mathrm{kpc}$ from the Galactic centre) are concentrated around this distance, the HB level should be related to the reddening by means of a slope $R_{\mathrm{V}}$. This is plotted in Figure 2, which is the updated version of Figure 5 in Barbuy et al. (1998). This analysis includes 12 clusters plus an arbitrary point at $E(B-V)=0$ and $V(\mathrm{HB})=15.18$, which should correspond to the $V(\mathrm{HB})$ at $8.0 \mathrm{kpc}$ from the Sun, with $[\mathrm{Fe} / \mathrm{H}]=-1.0, M_{\mathrm{V}}=0.8$, as explained above. Therefore, the best fit is $3.3<R_{\mathrm{V}}<3.1$. The higher slope of $R_{\mathrm{V}}=3.6$ seems too steep. This confirms that a choice of an average reddening value of $R_{\mathrm{V}}=3.2$ is adequate and that the barycentric distance from the Sun of the considered clusters should be around $8.0 \mathrm{kpc}$.

Finally, we address a few comments on the distance of UKS 1, since there is disagreement between different authors. We employed four sets of data: (a) NICMOS data (Ortolani et al. 2001, 2007), measured relative to NGC 6528, assumed to be at a distance of $d_{\odot}=7.7 \mathrm{kpc}$, results in $(m-M)_{\circ}=14.43$. Assuming that both clusters have a similar metallicity, and the $H S T / \mathrm{NICMOS}$ reddening law, we get $\mathrm{d}_{\odot}=15.8 \mathrm{kpc}$ for UKS 1; (b) NICMOS data measured relative to Liller 1 , assuming for Liller 1 a distance of $\mathrm{d}_{\odot}=8.1 \mathrm{kpc}$ (from $(m-M)_{\circ}=14.55$, Saracino et al. 2015); given a $\Delta(m-M)$ 。 $=1.02$, we get $(m-M)_{\circ}=15.57$ and a distance of $\mathrm{d}_{\odot}=$ 13.0 for UKS 1; (c) Assuming Minniti et al. (2011) absolute reference for the $\mathrm{HB}$ colour and magnitude $M_{\mathrm{K}}=-1.65$, $J-K=0.71$ (from a study of red clump calibrated stars with Hipparcos data), and using NICMOS calibrated data, we obtain $J_{\mathrm{HB}}=17.96$. From $M(\mathrm{HB})_{\mathrm{J}}=-0.94$ and $A_{\mathrm{J}}=$ 2.78, obtained from the comparison with Liller 1 (Saracino et al. 2015), we get $(m-M)_{\circ}=18.63-2.78=15.85$, and $\mathrm{d}_{\odot}$ $=14.8 \mathrm{kpc}$; (d) from Liller 1 (Saracino et al. 2015), but with a recalculated absolute distance from Minniti et al. (2011) values of $M(\mathrm{HB})_{\mathrm{K}}$ and $J-K(\mathrm{HB})$, we have: $(m-M)_{\circ}$ (Liller $1)=14.85\left(\mathrm{~d}_{\odot}=9.33 \mathrm{kpc}\right)$, and $(m-M)_{\circ}=14.85 \pm 1.02$ $=15.87$ and $\mathrm{d}_{\odot}=14.9 \mathrm{kpc}$ for UKS 1 . All these methods give an average of $\mathrm{d}_{\odot}=14.6 \mathrm{kpc}$ for UKS 1 , as reported in Table 2.

\section{CHEMICAL ABUNDANCES}

The metallicity distribution of bulge clusters as given in Table 1 is shown in Figure 4. It shows a peak around $[\mathrm{Fe} / \mathrm{H}]$ $\approx-1.0$, suggesting that this population is intrinsically significant. That such an old bulge stellar population is important, is confirmed by studies of RR Lyrae with the same metallicity, and corresponds to the lower end of the bulk of bulge stellar population metallicity distribution (see Sect. 2).

Table 3 shows the chemical abundances for a subset of GCs from Table 1, that have available high spectral resolution abundance analyses.

Carbon and Nitrogen: $\mathrm{C}$ and $\mathrm{N}$ show the expected anticorrelation due to $\mathrm{CNO}$ processing along the red giant branch. Two clusters with low $\mathrm{N}$ abundances should be further studied since this is not expected.

Odd-Z elements $\mathrm{Na}$, Al A crucial issue concerns possible $\mathrm{Na}-\mathrm{O}$ anticorrelation, which would indicate the presence of a second stellar generation. As a matter of fact, most GCs are presently being found to have at least two stellar generations, except possibly the least massive clusters. A threshold in mass for a second generation not to occur is presently estimated to be at 3-4 × $10^{4} \mathrm{M}_{\circ}$ (R.G. Gratton, private communication), or in other words, essentially all GCs should show the Na-O anticorrelation. The second generation is detected via $\mathrm{Na}-\mathrm{O}, \mathrm{Mg}-\mathrm{Al}$ anticorrelations, and also by the presence of both $\mathrm{CN}$-strong and $\mathrm{CN}$-weak stars (Carretta et al. 2009). The origin of these chemical anomalies is probably hot bottom burning (HBB) (Ventura et al. 2013) in massive Asymptotic Giant Branch (AGB) first generation stars, with yields ejected in the internal cluster medium, and incorporated by the second generation stars, the latter showing these anomalies. A thorough discussion on the origin of multiple stellar populations in GCs is given in Renzini et al. (2015). From Table 3, we see that very few of the bulge clusters were investigated in terms of $\mathrm{Na}$ and $\mathrm{Al}$. In fact, four stars analysed by Barbuy et al. (2014) show no Na-O anticorrelation (see their Figure 6), however, more stars have to be analysed for a firm conclusion. An important investigation on this matter was carried out by Gratton et al. (2015), where 17 BHB and 30 RHB stars of NGC 6723 were analysed. It was found that RHB and intermediate-BHB stars appear to belong to a first generation, showing O-rich and Na-poor abundances, whereas the bluest of the BHB stars show lower oxygen and higher $\mathrm{Na}$ (four $\mathrm{BHB}$ stars show $[\mathrm{O} / \mathrm{Fe}]=+0.23$ and $[\mathrm{Na} / \mathrm{Fe}]=+0.11)$, in contrast with the mean values given in 


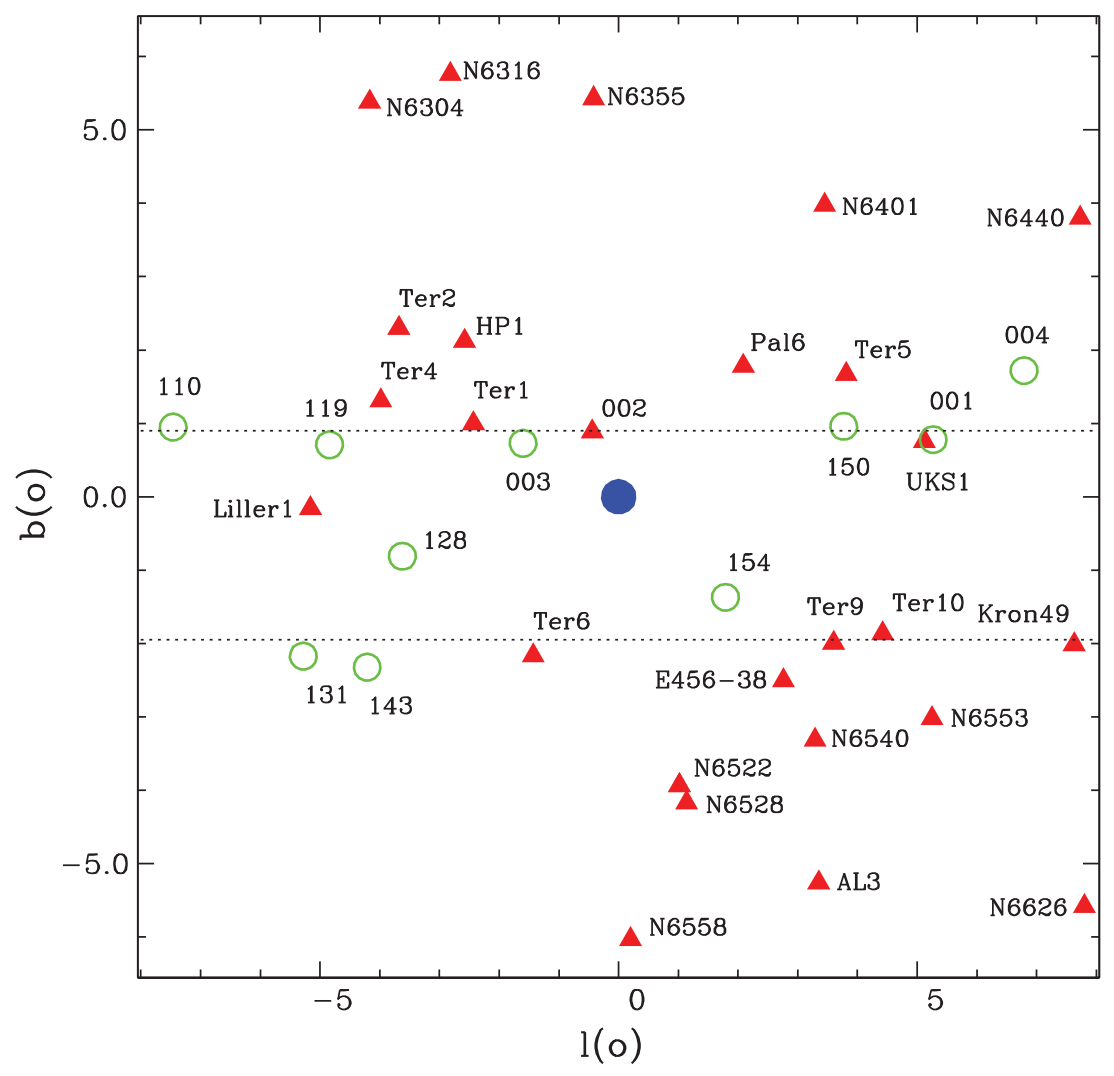

Figure 5. Location of central projected bulge clusters in Galactic coordinates. Symbols: redfilled triangles: bulge globular clusters (Table 1); green open circles: VVV clusters and candidates (Table 4). VVV clusters are identified by their numbers; blue-filled circle: Galactic centre; dotted lines encompass the so-called forbidden zone for optical globular clusters.

Table 3. Gratton et al. (2015) consider that extended blue HB stars might correspond to a second generation of lower mass stars.

Alpha-elements $\mathrm{O}, \mathrm{Mg}, \mathrm{Si}, \mathrm{Ca}, \mathrm{Ti}:$ In Figure 7 are plotted the abundances of these alpha elements versus $[\mathrm{Fe} / \mathrm{H}]$, including the abundances for the bulge sample, as given in Table 3, compared with abundances for 57 field bulge giant stars from Lecureur et al. (2007), Gonzalez et al. (2011), and Barbuy et al. (2015), and 58 bulge dwarf stars from Bensby et al. (2013). The oxygen abundances are as given in Barbuy et al. (2015), where they were revised with respect to Lecureur et al. (2007). Some discrepancy between the oxygen abundances from Barbuy et al. (2015) and Bensby et al. (2013) can be explained by the facts that: Barbuy et al. analysed red giants, using the forbidden [OI]6300.31 A line and Bensby et al. (2013) analysed dwarfs, using the permitted triplet OI lines at 7771.94, 7774.16, $7775.39 \AA$ A lines. Solar oxygen abundances adopted were respectively $\epsilon(\mathrm{O})=$ 8.87 and 8.85 for Barbuy et al. and Bensby et al., which would tend to invert the small shift between the two sets results. All in all, given that the permitted OI lines are wellknown to be subject to strong non-LTE effects, and tend to overestimate the oxygen abundances, we can consider that there is a good agreement between the two sets of oxygen abundances.
The panels in Figure 7 indicate therefore that the alphaelements $\mathrm{O}, \mathrm{Mg}, \mathrm{Si}$, and $\mathrm{Ca}$ are overabundant by about $[\mathrm{O}$, $\mathrm{Mg}, \mathrm{Si}, \mathrm{Ca}, \mathrm{Ti} / \mathrm{Fe}] \approx 0.3$ to $0.4 \mathrm{dex}$ for the more metalpoor stars. The same is found for the field (see also AlvesBrito et al. 2010; Bensby et al. 2013). This implies early fast enrichment by core collapse supernovae SNII, which in turn give a short timescale for bulge formation, where even the metal-rich clusters are old (Ortolani et al. 1995).

Heavy elements: Very few heavy element abundance derivation is available for individual stars of bulge GCs. The heavy elements of first peak Y, Sr, Zr, together with a few elements of the second peak Ba, La, and the $r$-element $\mathrm{Eu}$, can reveal the nature of the first stars, or else to reveal if AGBs were acting as important chemical contributors. A threshold of about $[\mathrm{Ba} / \mathrm{Eu}]=0.6$ would be indicative if $\mathrm{Ba}$ was produced by $r$ - or $s$-process. If $[\mathrm{Ba} / \mathrm{Eu}]>0.6$, this could be a hint of early enrichment by massive spinstars, and the same applies to the ratios $\mathrm{Y} / \mathrm{Ba}, \mathrm{Sr} / \mathrm{Ba}$, and $\mathrm{Zr} / \mathrm{Ba}$ (Frischknecht et al. 2016). These ratios were studied for the GC NGC 6522, by Barbuy et al. (2009), Chiappini et al. (2011), and Ness, Asplund, \& Casey (2014) using GIRAFFE spectra from the survey by Zoccali et al. (2008). Barbuy et al. (2014) used new UVES spectra observed in 2012 for four of the same stars, and these new results are reported in Table 3. Sr lines are unreliable as shown by Barbuy et al. (2014), and Ness 

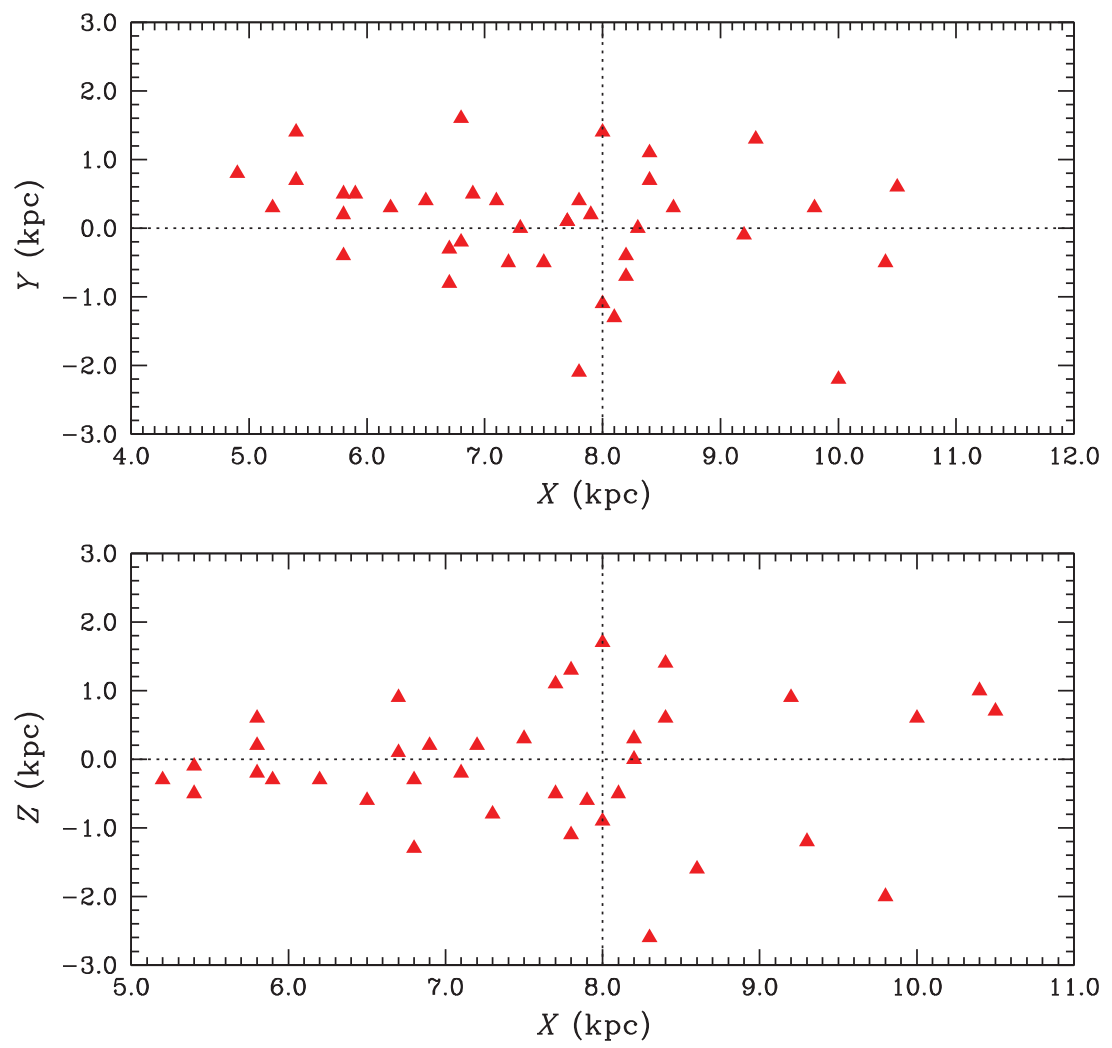

Figure 6. Bulge sample (Table 1). Upper panel: in the $X, Y$ plane; Lower panel: $X, Z$ plane.

et al. (2014), superseding abundance values given in Chiappini et al. (2011). Zr is difficult to derive: in Table 3, it is not reported given that it is only available for NGC 6522, but it is worth mentioning that Cantelli, Barbuy, Chiappini, Depagne, Pignatari, Hirschi, Ortolani, and Bica (in preparation) seems to detect a clear variation in $\mathrm{Zr}$ abundances among 12 member stars of NGC 6522 observed with GIRAFFE in 2012. The ratios from first to second peak of heavy elements can also be explained by production of $s$-process heavy elements in massive AGB stars, and predicted relative ratios between different heavy elements was presented in Bisterzo et al. (2010, 2014). Finally, it is possible that all heavy elements in old stars were produced by the $r$-process only, as first suggested by Truran (1981).

\section{KINEMATICS AND ORBITS}

According to Minniti \& Zoccali (2008), the bulge kinematics as viewed from the field stars lies between a purely rotational system and a velocity dispersion supported one. Another important feature of stellar kinematics is the presence of a massive bar in the bulge (Blitz \& Spergel 1991). An Xshape of the bulge related to the box/peanut configuration has been suggested by McWilliam \& Zoccali (2010) and Nataf et al. (2010), from the double red clump detected in the near-IR CMDs of the bulge fields. This is interpreted as secular evolution of bars (e.g. Athanassoula 2005) and leads to the idea that the galactic bulge is not a classical bulge. This issue is developed in other reviews in this volume. Observations of the X-shape profile are detailed thoroughly in Wegg, Gerhard, \& Portail (2015 and references therein). It is a generic feature of boxy bulges and is seen in many other galaxies (e.g. Bureau et al. 2006).

Even if the X-shape is a confirmed feature, it is interesting to point out a recent study by Lee, Joo, \& Chung (2015), that suggests an alternative explanation based on the presence of two different populations with a second generation of stars helium-enhanced and more metal rich, having a brighter clump than the first, more metal-poor component. In this model, there is no need for a deviation from a classical bulge shape. This is basically the same framework currently accepted for the multi-population features in the massive galactic GCs. Some issues are still open in this new interpretation, such as the source and the efficiency of the enrichment mechanism and the needed yields for the second generation component, in a wide environment such as the Galactic bulge. Accurate kinematics of the two clumps (for example, from $G A I A$ ) could disentangle between the two scenarios.

\subsection{Bulge field properties}

Kinematics. Babusiaux et al. (2010, 2014) carried out a kinematical study of 650 bulge field stars, and concluded that the more metal-poor stars, where a lower end at around $[\mathrm{Fe} / \mathrm{H}] \approx-1.0$ was found, correspond to a spheroidal distribution. The metal-rich stars showed instead a kinematics 
Table 3. Metallicities and abundances from high-resolution spectroscopy. References: 11: Origlia, Valenti, \& Rich 2005a; 12: Origlia \& Rich 2004; 13: Barbuy et al. 2006, 2015, 2014; 14: Origlia, Rich, \& Castro 2002; 15: Valenti et al. 2015; 16: Lee, Carney, \& Balachandran 2004; 17: Origlia et al. 2011; 18: Origlia, Valenti, \& Rich 2008; 19: Origlia et al. 2005b; 20: Barbuy et al. 2014; 21: Zoccali et al. 2004; 22: Carretta et al. 2001; 23: Meléndez et al. 2003 plus Origlia et al. 2002; 24: Cohen et al. 1999; 25: Alves-Brito et al. 2006; 26: Barbuy et al. 2007; 27: Valenti, Origlia, \& Rich 2011; 28: Smith \& Wehlau 1985; 29: Lee 2007; 30: Gratton et al. 2015 for BHB; 31 : Gratton et al. 2015 for RHB; 32: Peñaloza et al. 2015; 33 Rojas-Arriagada et al. 2016.

\begin{tabular}{|c|c|c|c|c|c|c|c|c|c|c|c|c|c|c|c|}
\hline Name & {$[\mathrm{Fe} / \mathrm{H}]$} & {$[\mathrm{C} / \mathrm{Fe}$} & {$[\mathrm{N} / \mathrm{Fe}]$} & {$[\mathrm{O} / \mathrm{Fe}]$} & {$[\mathrm{Na} / \mathrm{Fe}]$} & {$[\mathrm{Al} / \mathrm{Fe}]$} & {$[\mathrm{Mg} / \mathrm{Fe}]$} & {$[\mathrm{Si} / \mathrm{Fe}]$} & {$[\mathrm{Ca} / \mathrm{Fe}]$} & {$[\mathrm{Ti} / \mathrm{Fe}]$} & {$[\mathrm{Y} / \mathrm{Fe}]$} & {$[\mathrm{Ba} / \mathrm{Fe}]$} & {$[\mathrm{La} / \mathrm{Fe}]$} & {$[\mathrm{Eu} / \mathrm{Fe}]$} & ref. \\
\hline NGC 6342 & -0.60 & -0.34 & - & +0.31 & - & - & +0.38 & +0.37 & +0.38 & +0.25 & - & - & - & 11 & \\
\hline Terzan 4 & -1.60 & -0.25 & - & +0.54 & - & - & +0.41 & +0.55 & +0.54 & +0.44 & - & - & - & - & 12 \\
\hline HP 1 & -1.00 & - & - & +0.40 & +0.00 & - & +0.10 & +0.30 & +0.03 & +0.02 & - & +0.15 & +0.00 & +0.15 & 13 \\
\hline Liller 1 & -0.30 & - & - & - & - & - & +0.3 & +0.3 & +0.3 & - & - & - & - & - & 14 \\
\hline Terzan 1 & -1.26 & - & - & +0.39 & - & - & +0.42 & +0.31 & - & +0.15 & - & - & - & - & 15 \\
\hline Pal 6 & -1.00 & - & - & - & - & - & - & +0.40 & - & +0.50 & - & - & - & - & 16 \\
\hline Terzan 5 & -0.25 & -0.34 & - & +0.28 & - & - & +0.30 & +0 & +0.31 & +0.31 & - & - & - & - & 12 \\
\hline Terzan 5 & 0.2 & -0.40 & - & -0.05 & - & - & +0 & & & +0.05 & - & - & - & - & 17 \\
\hline NGC 6440 & -0.50 & -0.57 & +0.33 & - & +0.46 & +0.33 & & & & - & - & - & - & - & 18 \\
\hline UKS 1 & -0.78 & -0.45 & - & +0.28 & - & - & +0 & +0 & & +0.32 & - & - & - & - & 19 \\
\hline NGC 6522 & -0.95 & -0.03 & +0.67 & +0.36 & -0.07 & -0.11 & +0.23 & +0.13 & +0.13 & +0.10 & +0.31 & +0.02 & -0.01 & -0.14 & 20 \\
\hline NGC 6528 & -0.17 & -0.11 & -- & +0.15 & +0.43 & - & +0.07 & +0.08 & -0.40 & -0.10 & - & - & - & - & 21 \\
\hline NGC 6528 & +0.07 & - & - & +0.07 & +0.40 & - & +0.14 & +0.36 & +0.23 & +0.03 & - & +0.14 & - & - & 22 \\
\hline NGC 6528 & -0.17 & -0.35 & - & +0.33 & - & - & +0.35 & +0.30 & +0.37 & +0.31 & - & - & - & - & 11 \\
\hline NGC 6539 & -0.76 & -0.30 & - & +0.41 & - & - & +0.45 & +0.43 & +0.43 & +0.42 & - & - & - & - & 19 \\
\hline NGC 6553 & $-0.20^{4}$ & $-0.08^{4}$ & $+0.30^{4}$ & $+0.43^{4}$ & - & - & $+0.3^{5}$ & $+0.3^{5}$ & $+0.3^{5}$ & - & - & - & - & - & 23 \\
\hline NGC 6553 & -0.17 & - & - & +0.50 & - & - & +0.41 & +0.14 & +0.26 & +0.19 & - & - & - & - & 24 \\
\hline NGC 6553 & $-0.21^{4}$ & - & - & - & +0.16 & +0.18 & +0.28 & +0.21 & +0.05 & -0.01 & - & -0.28 & -0.11 & +0.10 & 25 \\
\hline NGC 6558 & -0.97 & - & - & +0.38 & -0.09 & +0.02 & +0.24 & +0.23 & +0.05 & +0.06 & - & +0.13 & 0.00 & +0.36 & 26 \\
\hline Mercer 5 & -0.85 & - & - & +0.31 & - & - & - & & - & +0 & - & - & - & - & 32 \\
\hline NGC 6624 & -0.69 & -0.29 & - & +0.41 & - & +0.39 & +0.42 & +0.38 & +0.40 & +0.37 & - & - & - & - & 27 \\
\hline NGC 6626 & -1.00 & - & - & - & - & - & - & - & - & - & - & - & - & - & 28 \\
\hline NGC 6637 & -0.77 & - & - & +0.20 & +0.35 & +0.49 & +0.28 & +0.45 & +0.20 & +0.24 & +0.13 & +0.22 & +0.21 & +0.45 & 29 \\
\hline NGC 6723 & -1.22 & - & +0.85 & +0.39 & +0.04 & - & +0.52 & - & - & - & - & - & - & - & 30 \\
\hline NGC 6723 & -1.22 & - & - & +0.55 & +0.11 & - & +0.50 & +0.59 & +0.81 & - & - & +0.75 & - & - & 31 \\
\hline NGC 6723 & -0.98 & - & - & +0.28 & +0.00 & +0.31 & +0.23 & +0.36 & +0.30 & +0.24 & - & +0.22 & - & - & 33 \\
\hline
\end{tabular}

typical of the bar (see also review by Gonzalez \& Gadotti 2016). Vásquez et al. (2013) studied the kinematics of 454 field bulge stars located in the bright and the faint red clumps of the X-shaped bulge. They conclude that stars with elongated orbits tend to be metal-poor, whereas the metal-rich ones are preferentially in axisymmetric orbits, at odds with conclusions by Babusiaux et al. (2010).

Ages. Clarkson et al. (2008, 2011) used proper motion cleaned data, deriving a cleaned bulge CMD, demonstrated to consist of an old population of at least $10 \mathrm{Gyr}$. The same conclusion had been reached previously by Zoccali et al. (2003).

\subsection{Kinematics and orbits of bulge GCs}

Space velocities for the GCs, that require radial velocities and proper motions to be derived, are only available for part of the sample. Tables 1 and 2 gather space velocities with respect to the Sun, and corresponding references. These space velocities, which result from a combination of radial velocities and proper motions, are reported in column 12 of Table 1 and column 8 of Table 2. Earlier work was carried out by Dinescu et al. (1997, 1999a,1999b, 2003) and Casetti-Dinescu et al. (2007, 2010, 2013). Using HST data, Zoccali et al. (2003) and
Feltzing \& Johnson (2002) derived space velocities for the metal-rich clusters NGC 6553 and NGC 6528, respectively. Rossi et al. (2015) measured proper motion cleaned CMDs from long time base data, and derived space velocities for 10 central GCs.

An important piece of information was revealed by the orbits of the inner GCs derived by Rossi et al. (in preparation): all GCs located in the inner bulge appear to be trapped in the bar (Rossi et al. 2015; Rossi et al., in preparation). We point out that the clusters, in particular, the moderately metal-poor ones, probably formed very early in the very central parts of the Galaxy (e.g. NGC 6522, NGC 6558), before the bar instability occurred. This is confirmed by their rotational velocity counter-rotating with respect to the bar and the Galaxy in some cases. Since these clusters have low heights $z$, their retrograde orbits can be considered as robust with respect to variations of the bar shape (Pfenniger 1984). Therefore, it seems that whenever the bar formed, given their low kinematics, essentially all GCs would be trapped. Martinez-Valpuesta, Shlosman, \& Heller (2006) suggested that a first vertical buckling of the growing bar occurred at 1.8-2.8 Gyr, and a second at 6-7.5 Gyr. The bar then assumes a boxy or peanut X-shape. The trapping of GCs in the boxy bulge includes bulge clusters of all metallicities, from 

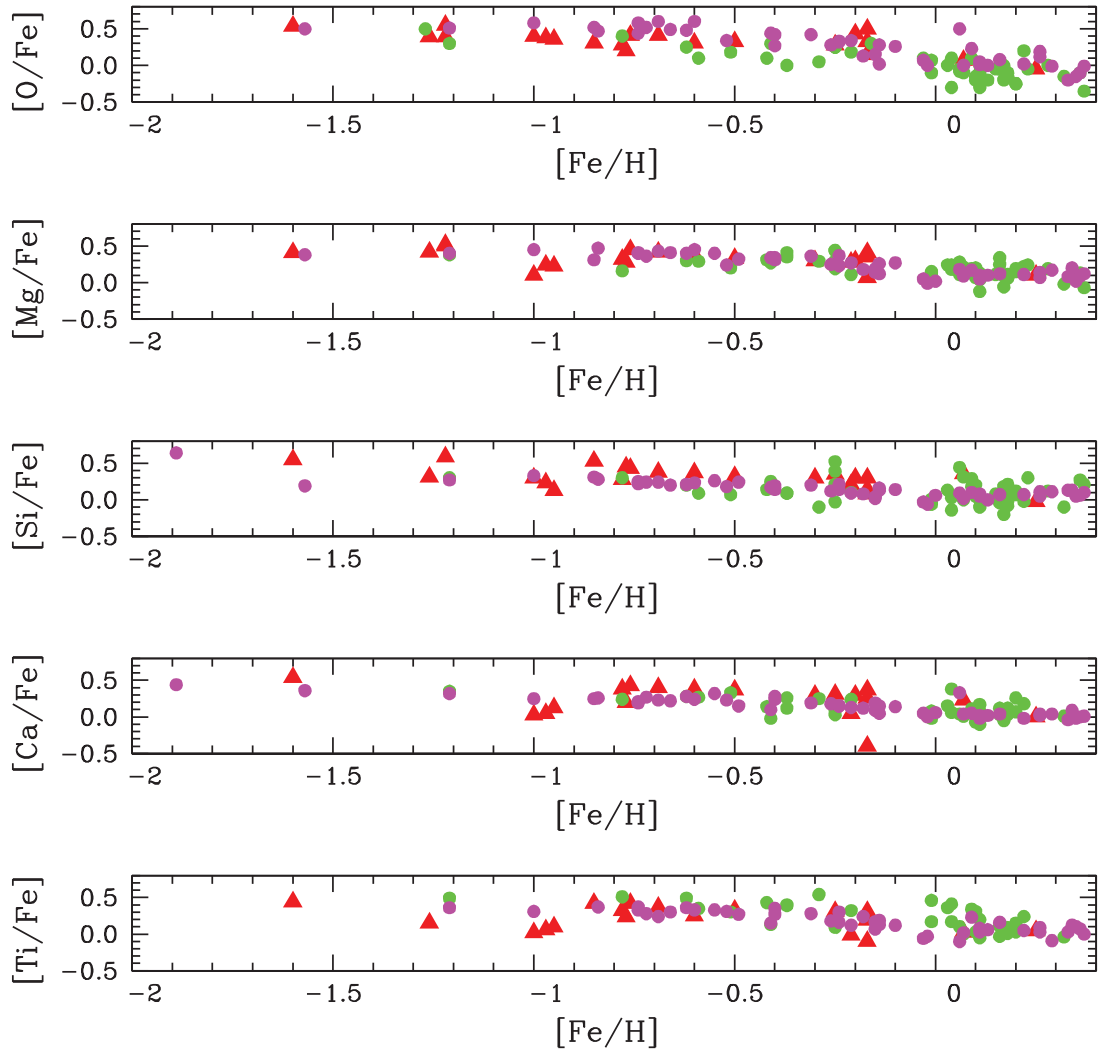

Figure 7. $[\mathrm{O}, \mathrm{Mg}, \mathrm{Si}, \mathrm{Ca}, \mathrm{Ti} / \mathrm{Fe}]$ versus $[\mathrm{Fe} / \mathrm{H}]$ for sample globular clusters, compared with field stars. Symbols: red triangles: sample globular clusters from Table 3; green circles: 56 bulge giants analysed by Lecureur et al. (2007), Barbuy et al. (2015) and Gonzalez et al. (2011); magenta circles: microlensed bulge dwarfs analysed by Bensby et al. (2013). For Terzan 5, NGC 6528, NGC 6553, and NGC 6723, 2, 3, 3, and 2 sets of values are included, respectively.

moderately metal-poor as mentioned above, to metal-rich ones like Terzan 2.

A main conclusion is that the inner clusters are confined and possibly trapped in the bar, due to the high mass of the bar, achievable due to the low velocity components of the clusters.

\section{FUTURE STEPS}

Figure 5 shows the GCs projected in the central $\|1\|<8^{\circ}$ and $\|\mathrm{b}\|<5^{\circ}$. These include the sample by Barbuy et al. (1998), which was given in a radius of $R<5^{\circ}$, and a few more, in particular, Terzan 9, $\mathrm{Al} 3$, and results from the VVV survey. The VVV survey provided new GCs and candidates in that region (Borissova et al. 2011, 2014). The clusters VVV CL001, CL 002, CL 003, and CL 004 were studied by means of the VVV photometric catalogue (Minniti et al. 2011, Moni Bidin et al. 2011). VVV CL 002 appears to be the most central GC. VVV CL 001 is projected very close to UKS 1 and they may be a binary system (Minniti et al. 2011). In this case, UKS 1 might be interpreted as a dwarf galaxy remains, similarly to Terzan 5. VVV CL 003 seems to be a far side GC or old open cluster, while VVV CL 004 might be rather an old open cluster. Table 4 shows available information on the VVV sample, including ages and GC candidates (Borissova et al. 2014). Table 4 reveals a number of candidates in zones where 2MASS and GLIMPSE could not detect clusters. These previous surveys showed GCs outside the central bulge only.

Figure 5 shows the angular distribution of known GCs projected in the central parts of the Galaxy, and candidate ones found in the VVV survey. This figure, together with Table 1, clearly show the depletion of GCs on the far side. Besides, VVV CL 002 is very close to the Galactic centre, and VVV CL 003 is in the far size. The VVV candidates (Table 4) may mitigate that asymmetric distribution, but will not completely solve the problem of missing GCs in the far side. We recall, however, that when IR distances are taken into account, the clusters are well distributed around $8 \mathrm{kpc}$ (Section 3).

Ivanov, Kurtev, \& Borissova (2005) estimated that in the central parts of the Galaxy at least $10 \pm 3$ GCs were missing. Several new GCs have been discovered or identified as such in the last decade, especially with observations in the near IR, in the area outside Figure 5, and recently, in the inner bulge region VVV CL 002 has been added. The VVV candidates are a promising sample to further populate the inner bulge. 
Table 4. VVV GCs and candidates. References: 1: Minniti et al. (2011); 2: Moni Bidin et al. (2011); 3: Borissova et al. (2014).

\begin{tabular}{|c|c|c|c|c|c|c|c|}
\hline $\begin{array}{l}\text { VVV } \\
1\end{array}$ & $\begin{array}{c}1 \\
(0) \\
2\end{array}$ & $\begin{array}{c}\mathrm{b} \\
(\mathrm{o}) \\
3\end{array}$ & $\begin{array}{c}\text { Alpha } \\
\text { h:m:s } \\
4\end{array}$ & $\begin{array}{c}\text { Delta } \\
\text { (o): :'” } \\
5\end{array}$ & $\begin{array}{c}\text { Appr.size } \\
\text { (') } \\
6\end{array}$ & $\begin{array}{c}\text { Comments } \\
7\end{array}$ & $\begin{array}{c}\text { References } \\
8\end{array}$ \\
\hline CL110 & 352.54 & 0.95 & $17: 22: 47$ & $-34: 41: 17$ & 0.4 & GC/old OC & 3 \\
\hline CL119 & 355.16 & 0.71 & $17: 30: 46$ & $-32: 39: 05$ & 1.8 & old OC & 2 \\
\hline CLO03 & 358.40 & 0.73 & $17: 38: 55$ & $-29: 54: 25$ & 2.2 & old OC/GC,far side(13 kpc) & 2 \\
\hline CL128 & 356.38 & -0.81 & $17: 39: 59$ & $-32: 26: 27$ & 1.0 & GC cand./Old OC & 3 \\
\hline CLO02 & 359.56 & 0.89 & $17: 41: 06$ & $-28: 50: 42$ & 3.0 & GC & 2 \\
\hline CL131 & 354.72 & -2.17 & $17: 41: 17$ & $-34: 34: 02$ & 1.7 & old OC/GC cand. & 3 \\
\hline CL143 & 355.79 & -2.32 & $17: 44: 36$ & $-33: 44: 18$ & 1.3 & old OC/young GC & 2 \\
\hline CL150 & 3.77 & 0.96 & $17: 50: 41$ & $-25: 13: 06$ & 0.8 & old OC/young GC,10Gyr & 3 \\
\hline CL154 & 1.79 & -1.37 & $17: 55: 08$ & $-28: 06: 01$ & 0.7 & old OC, 8 Gyr & 3 \\
\hline CLO04 & 6.79 & 1.72 & $17: 54: 32$ & $-22: 13: 38$ & 1.6 & old OC? & 2 \\
\hline CLO01 & 5.27 & 0.78 & $17: 54: 43$ & $-24: 00: 53$ & 0.5 & GC, 8 from UKS 1 & 1 \\
\hline
\end{tabular}

Figure 5 portrays the current status of the inner bulge sample, together with VVV GCs and candidates (Table 1). As pointed out by Barbuy et al. (1998), a zone of avoidance in the GC distribution occurs for $0.9<\mathrm{b}<-1.9$. It is related to dust heavily absorbing in the disk and/or dynamical effects on the GC population by the disk (bar) and bulge. The zone of avoidance is asymmetric in Galactic latitude because of the Sun's offset of about 18 pc above the Galactic plane. The zone of avoidance now includes 5 VVV candidates (Figure 5), while the other ones are distributed as in the sample available previously in Barbuy et al. (1998).

Finally, as concerns a possible binarity between VVV CL 001 and UKS 1 , it is interesting to note that, likewise, the halo dwarf spheroidal Ursa Minor has a GC companion (Muñoz et al. 2012). Growing evidence suggests that a fraction of the inner bulge sample are galaxy remains. In the coming years, Gaia may show streamers related to them, to further constrain the dynamical issues involved.

\section{CONCLUSIONS}

In this review, we provide a state-of-the-art list of bulge GCs and their properties. We tried to constrain their properties including their kinematics when available. Kinematics is becoming a key tool to identify their nature, and the link with the field stellar populations, within the complex substructures of the bulge.

In recent years, good progress in the knowledge of the GCs in the central parts of the Galaxy has been achieved. For spectroscopy, the multi-object spectrographs in $8 \mathrm{~m}$ class telescopes have made it possible to derive chemical abundances for considerable numbers of stars. Progress in instrumentation thanks to imaging with MCAO in the infrared (MAD/VLT, GEMS/Gemini) and HST/NICMOS, has made possible long time baseline of CCD data with excellent quality, allowing proper motion cleaning of CMDs. Finally, surveys with larger apertures such as the $4 \mathrm{~m}$ VISTA Telescope provided the discovery of new objects. The VVV survey in particular has provided several new bulge globular clusters and candidates, to be explored in coming years.

Much work is still needed as concerns bulge globular clusters, such as the monitoring of variable stars, in particular RR Lyrae, to obtain deep CMDs allowing age derivation, and the derivation of metallicities and chemical abundances from high-resolution spectroscopy. The derivation of $\mathrm{Na}$ and $\mathrm{O}$ abundances will be crucial to define if these very old clusters have a unique or multiple stellar generations. These results should allow to better compare the bulge globular clusters with outer bulge, inner halo, and outer halo ones.

\section{ACKNOWLEDGEMENTS}

$\mathrm{EB}$ and $\mathrm{BB}$ acknowledge partial financial support from $\mathrm{CNPq}$, CAPES, and Fapesp. SO acknowledges the Italian Ministero dell'Università e della Ricerca Scientifica e Tecnologica (MURST), Italy.

\section{REFERENCES}

Alves-Brito, A., et al. 2006, A\&A, 460, 269

Alves-Brito, A., Meléndez, J., Asplund, M., Ramírez, I., \& Yong, D. 2010, A\&A, 513, A35

Armandroff, T. 1989, AJ, 97, 375

Athanassoula, E. 2005, MNRAS, 358, 1477

Baade, W. 1944, ApJ, 100, 137

Baade, W. 1946, PASP, 343, 249

Babusiaux, C., et al. 2010, A\&A, 519, 77

Babusiaux, C., et al. 2014, A\&A, 563, 15

Barbuy, B., Bica, E., \& Ortolani, S. 1998, A\&A, 333, 117

Barbuy, B., et al. 2014, A\&A, 570, 76

Barbuy, B., et al. 2015, A\&A, 580, 40

Barbuy, B., et al. 2006, A\&A, 449, 349

Barbuy, B., et al. 2007, AJ, 134, 1613

Barbuy, B., et al. 2009, A\&A, 507, 405

Bechtol, K., et al. 2015, ApJ, 807, 50

Bensby, T., et al. 2013, A\&A, 549, 147

Bica, E., Bonatto, C., Barbuy, B., \& Ortolani, S. 2006, A\&A, 450, 105 
Bica, E., \& Pastoriza, M. 1983, ApSSci, 91,99

Bisterzo, S., et al. 2010, MNRAS, 404, 1529

Bisterzo, S., et al. 2014, ApJ, 787, 10

Blitz, L., \& Spergel, D. N. 1991, ApJ, 379, 631

Bobylev, V. V., Mosenkov, A. V., Bajkova, A. T., \& Gontcharov, G. A. 2014, AstL, 40, 86

Bonatto, C., Bica, E., Ortolani, S., \& Barbuy, B. 2007, MNRAS, 381, L45

Bonatto, C., Bica, E., Ortolani, S., \& Barbuy, B. 2009, MNRAS, 397, 1032

Borissova, J., et al. 2014, A\&A, 569, 24

Borissova, J., et al. 2011, A\&A, 532, 131

Bureau, M., et al. 2006, MNRAS, 370, 753

Cannon, A. J. 1929, BHarO, 868, 1

Cardelli, J. A., Clayton, G. C., \& Mathis, J. S. 1989, ApJ, 345, 245

Carretta, E., et al. 2009, A\&A, 505, 117

Carretta, E., et al. 2010, A\&A, 516, 55

Carretta, E., Cohen, J. G., Gratton, R. G., \& Behr, B. B. 2001, AJ, 122,1469

Casetti-Dinescu, D. I., et al. 2007, AJ, 134, 195

Casetti-Dinescu, D. I., et al. 2013, AJ, 146, 33

Casetti-Dinescu, D. I., et al. 2010, AJ, 140, 1282

Clarkson, W., et al. 2008, ApJ, 684, 1110

Clarkson, W., et al. 2011, ApJ, 735, 37

Chiappini, C., et al. 2011, Nature, 472, 454

Cohen, J. G., Gratton, R. G., Behr, B. B., \& Carretta, E. 1999, ApJ, 523, 739

Côté, P. 1999, AJ, 118, 406

Courtes, G., \& Cruvellier, P. 1960, POHP, 5, 3

Cudworth, K. M., \& Hanson, R. B. 1993, AJ, 105, 168

Dean, J. F., Warren, P. R., \& Cousins, A. J. 1978, MNRAS, 183, 569

Dékány, I., et al. 2013, ApJ, 776, L19

Dinescu, D. I., Girard, T. M., \& van Altena, W. F. 1997, AJ, 114, 1014

Dinescu, D. I., van Altena, W. F., \& Girard, T. M. 1999a, AJ, 117, 277

Dinescu, D. I., Girard, T. M., \& van Altena, W. F. 1999b, AJ, 117, 1792

Dinescu, D. I., Girard, T. M., van Altena, W. F., \& López, C. E. 2003, AJ, 125, 1373

Feltzing, S., \& Johnson, R. A. 2002, A\&A, 385, 67

Ferraro, F. R., et al. 2009, Nature, 462, 483

Fitzpatrick, E. L. 1999, PASP, 111, 63

Frenk, C. S., \& White, S. D. M. 1982, MNRAS, 198, 173

Frischknecht, U., et al. 2016, MNRAS, 453, 1803

Gran, F., et al. 2016, (arXiv:1604.01336)

Gratton, R. G., et al. 2015, A\&A, 573, A92

Grebel, E. K., \& Roberts, W. J. 1995, A\&AS, 109, 293

Gonzalez, O. A., \& Gadotti, D. A. 2016, in Astrophysics and Space Science Library, Galactic Bulges, ed. E. Laurikainen, R. Peletier, D. Gadotti (Vol. 418; Switzerland: Springer International Publishing), 199

Gonzalez, O. A., et al. 2011, A\&A, 530, 54

Harris, W. E. 1996, AJ, 112, 1487

Harris, W. E. 2001, in Star Clusters, Saas-Fee Advanced Courses, ed. L. Labhardt, \& B. Binggeli (Vol. 28; Berlin Heidelberg: Springer-Verlag), 223, ISBN 978-3-540-67646-1.

Hendricks, B., Stetson, P. B., VandenBerg, D. A., \& Dall'Ora, M. 2012, ApJ, 144, 25

Hill, V., et al. 2011, A\&A, 534, 80
Ivanov, V. D., Kurtev, R., \& Borissova, J. 2005, A\&A, 442, 195

Jones, R. V., Carney, B. W., Storm, J., \& Latham, D. W. 1992, ApJ, 386, 646

Kinman, T. D. 1959, MNRAS, 119, 538

Lecureur, A., et al. 2007, A\&A, 465, 799

Lee, J. W. 2007, RevMexAA, 28, 120

Lee, J.-W., Carney, B. W., Balachandran, S. C. 2004, AJ, 128, 2388

Lee, Y.-W. 1992, AJ, 104, 1780

Lee, Y.-W., Joo, S.-J., \& Chung, C. 2015, MNRAS, 453, 3906

Martinez-Valpuesta, I., Shlosman, I., \& Heller, C. 2006, ApJ, 637, 214

Massari, D., et al. 2014, ApJ, 795, 22

Mayall, M. U. 1946, ApJ, 104, 290

McCall, M. 2004, AJ, 128, 2144

McClure, R. D. 1969, AJ, 74, 50

McConnachie, A. W. 2012, AJ, 144, 4

McWilliam, A., \& Zoccali, M. 2010, ApJ, 724, 1491

Meléndez, J., et al. 2003, A\&A, 411, 417

Minniti, D. 1995, AJ, 109, 1663

Minniti, D., et al. 2011, A\&A, 527, 81

Minniti, D., \& Zoccali, M. 2008, IAU Symp., 245, 323

Moni Bidin, C., et al. 2011, A\&A, 535, 33

Morgan, W. W. 1959, AJ, 64, 432

Muñoz, R. R., et al. 2012, ApJ, 753, L15

Nataf, D. M., Udalski, A., Gould, A., Fouqué, P., \& Stanek, K. Z. 2010, ApJ, 721, L28

Ness, M., Asplund, M., \& Casey, A. R. 2014, MNRAS, 445, 2994

Ness, M., Freeman, K., Athanassoula, E., et al. 2013a, MNRAS, 432, 2092

Ness, M., Freeman, K., Athanassoula, E., et al. 2013b, MNRAS, 430, 836

Olson, B. I. 1975, PASP, 87, 349

Origlia, L., et al. 2013, ApJ, 779, L5

Origlia, L., Rich, R. M., \& Castro, S. 2002, AJ, 123, 1559

Origlia, L., et al. 2011, ApJ, 726, L20

Origlia, L., \& Rich, R. M. 2004, AJ, 127, 3422

Origlia, L., Valenti, E., \& Rich, R. M. 2005a, MNRAS, 356, 1276

Origlia, L., Valenti, E., Rich, R. M., \& Ferraro, F. 2005b, MNRAS, 363, 897

Origlia, L., Valenti, E., \& Rich, R. M. 2008, MNRAS, 388, 1419

Ortolani, S., Bica, E., \& Barbuy, B. 1999, A\&AS, 138, 267

Ortolani, S., Barbuy, B., Bica, E., Zoccali, M., \& Renzini, A. 2007, A\&A, 470, 1043

Ortolani, S., et al. 2001, A\&A, 376, 878

Ortolani, S., et al. 2011, ApJ, 737, 31

Ortolani, S., Bonatto, C., Bica, E., \& Barbuy, B. 2009, AJ, 138, 889

Ortolani, S., et al. 2012, AJ, 144, 147

Ortolani, S., et al. 1995, Nature, 377, 701

Peñaloza, F., et al. 2015, PASP, 127, 329

Pfenniger, D. 1984, A\&A, 134, 373

Pietrukowicz, P., et al. 2015, ArXiv:1412.4121

Pietrukowicz, P., et al. 2012, ApJ, 750, 169

Racine, R., \& Harris, W. E. 1989, AJ, 98, 1609

Reid, M. J., et al., 2014, ApJ, 783, 130

Renzini, A., et al. 2015, MNRAS, submitted

Rich, R. M. 2013, in Planets, Stars and Stellar Systems, ed. T. D. Oswal, \& G. Gilmore, (Vol. 5; Dordrecht: Springer Science+Business Media), 271, ISBN 978-94-007-5611-3.

Rojas-Arriagada, A., et al. 2014, A\&A, 569, 103

Rojas-Arriagada, A., et al. 2016, A\&A, 587, 95

Rossi, L. 2015, MNRAS, 450, 3270 
Saracino, S., et al. 2015, ApJ, 806, 152

Smith, H. A., \& Wehlau, A. 1985, ApJ, 298, 572

Terzan, A. 1968, POHP, 10, 4

Truran, J. 1981, A\&A, 97, 391

Valenti, E., Ferraro, F., \& Origlia, L. 2007, AJ, 133, 1287

Valenti, E., Origlia, L., Mucciarelli, A., \& Rich, R. M. 2015, A\&A, 574,80

Valenti, E., Origlia, L., \& Rich, R. M. 2011, MNRAS, 414, 2690

van den Bergh, S. 1967, AJ, 72, 70

Vásquez, S., et al. 2013, A\&A, 555, 91
Ventura, P., Di Criscienzo, M., Carini, R., \& D’Antona, F. 2013 , MNRAS, 431, 3642

Webbink, R. F. 1985, IAU Symp., 113, 541

Wegg, C., Gerhard, O., \& Portail, M. 2015, MNRAS, 450, 4050

Whitford, A. E., \& Rich, R. M. 1983, ApJ, 274, 723

Zinn, R. 1985, ApJ, 293, 424

Zoccali, M., et al. 2004, A\&A, 423, 507

Zoccali, M., et al. 2008, A\&A, 486, 177

Zoccali, M., et al. 2001, AJ, 121, 2638

Zoccali, M., et al. 2003, A\&A, 399, 931 